\title{
Introducing Collaborative Practices in Small Medium Enterprises
}

\author{
D. Antonelli, P. Chiabert
}

\author{
Dario Antonelli, Paolo Chiabert \\ Politecnico di Torino \\ Department of Production Systems and Economics \\ Corso Duca degli Abruzzi 24, 10129 - Torino, Italy \\ E-mail: \{dario.antonelli,paolo.chiabert\}@ polito.it
}

\begin{abstract}
In an enterprise, collaborative working schemes are obtained only by adopting a suitable organization of the enterprise functions. At the informative level collaboration can be enabled by using suitable project management tools for the exchange of information that is the basis of collaboration. One of these tools is Product Lifecycle Management (PLM). On the basis of an exhaustive analysis of the PLM impact on the different aspects of the enterprise, the paper proposes an original methodology to evaluate the possibility of using PLM as framework in which exploit collaboration links within the enterprise. The methodology is not founded on the formal declared organization but on the actual data flows that are induced by the relations among the documents used to develop a project. Data are collected on the field using questionnaires. The links are made explicit by applying the hierarchical clustering with single linkage agglomerative technique. The attitude to the implementation of PLM is then analysed with respect to the organization and to the informative system. Such methodology is general and can be profitably applied to assist the PLM implementation in the enterprises.
\end{abstract}

Keywords: Product Life-Cycle Management, Concurrent Engineering, Business Process Management, Cluster analysis, Collaboration.

\section{Introduction}

Small and Medium Enterprises (SMEs) are companies with a turnover of less than 50MEuro and a number of employees below 250, as for the EU Recommendation 2003/361/EC. In the European countries they are the backbone of the economic system [7]. Concurrent Engineering (CE) is an effective strategy to shorten 'time to market' of innovative products. The key for its accomplishment stays in the collaboration among all the enterprise personnel appointed to the project. The goal is accomplished by the parallel development of different activities obtained by the integration of several company functions in a multidisciplinary group in order to anticipate the constraints generated by the industrial dynamics [14]. The core aspect of CE is therefore the creation of tight co-operation links among all the industrial actors involved in the definition of product and process. A significant tool to assist the CE effectiveness is PLM. Unfortunately, PLM implementation inside SMEs is scarce. The reason goes beyond implementation difficulties or excessive costs of IT systems. It derives from the poor conformity of the ordinary SME organization of work to the requirements of CE in term of strategy, organization and technology [13].

It is useful to remark that often in literature the term collaboration is given the same significance as the term coordination. Collaboration means working with the others for the success of an activity. Coordination can refer to optimisation of the work by finding the best division of activities to be performed by humans and machines. The definition put forth by Malone and Crowston [15] is: "Coordination is managing dependencies between activities". Coordination also, recalling the theory of systems, implies the presence of a coordinator, then some hierarchical shape on the organisation [18]. Instead, collaboration is the process of various individuals, groups or systems working together on a voluntary basis without the need for a manager or a work program [16]. Obviously collaboration works only if some behaviour rules are respected, as the ones proposed in [21] in the case of collaborative software agents.

In the organization oriented to CE, different coordination mechanisms have been adopted [10]. They differ mainly on the instant of application of the coordination: predetermined before starting the work or determined during the work as a result of a negotiation. A discussed list of co-ordination systems is given in [20]: Information workflow, 
Negotiated objectives, Milestones, Professional rules, Mapped parameters, Design space sharing, Joint parameters, Data coherency. Some coordination systems need the active participation of a coordinator, e.g. the project manager. Conversely other systems like Design space sharing and Data coherency must be enforced by the use of appropriate software tools, e.g. the PLM.

A comprehensive definition of Product Lifecycle Management is "a strategic business approach for the effective management and use of corporate intellectual capital" where "corporate intellectual capital" consists of products definition, products history and best practices" [6]. Several PLM systems already operates in large companies demonstrating their effectiveness. They range from the Ford project C3P (CAD/CAE/CAM and Product Information Management) started in 1996 and based on SDRC's I-DEAS and Metaphase software to the Boeing 7E7 Dreamlinear airplane entirely designed in the Dassault Systemes PLM software platform, to the Siemens Teamcenter Suite largely adopted in automotive industries. Presently new lighter software suites, based on the "out of the shelf" commercial policy, make PLM systems affordable to SMEs. Despite the availability of software, PLM do not have an adequate diffusion among them.

In authors' opinion the most important justification of enterprises' interest in PLM should be the competitive edge provided by collaboration. As a matter of fact, PLM assures the most effective control of enterprise's projects by increasing the efficiency in information management along three directions:

- Increase of efficiency in the management of technical product information. Product data represent the core of manufacturing enterprise. They should be protected against unauthorized accesses but, at the same time, should be promptly shared for design activities as well as for many other functions in the enterprise.

- Easiness of product data exchange by establishing proper interfaces towards enterprise functions (manufacturing, maintenance ...) and, vice versa, by defining communicational channels to gather any relevant information for the upgrading.

- Management of the enterprise knowledge database for storing and retrieving historical products. The carry over process, widely adopted to speed up the time to market of new products, benefits from the use of PLM tools.

The reasons of its inadequate diffusion are uniformly spread over enterprise organization and information technology system:

- Accessing product data from enterprise functions not directly involved in product development is a functionality provided by IT tools like ERP that are seldom present inside the SMEs.

- Product/process development is historically a self-governed function, with human resources, activities and hardware/software tools sharply separated by other industrial functions.

- The implementation of IT tools linking different enterprises areas requires a clear definition of decisional and informational processes.

The idea behind this research is that the work organization inside a SME is based on spontaneous collaboration that overcomes the attempts to organize the activities by introducing coordination mechanisms, as in the case of PLM implementation. Spontaneous collaboration works well as far as the dimensions of the firm are small enough to allow everyone to know where to find the information they need. As far as the enterprise grows a technology like PLM becomes necessary to assist the information retrieval. To assess the validity of this supposition we need to find a way to measure the extent of collaboration. The paper illustrates an original approach to the analysis of management processes of SME's product data. Using simple data-driven procedures, it is possible to forecast the impact of collaboration on SME organization without inferring formal models in any case available in literature [4]. In detail, the proposed analysis evaluates the SME readiness to PLM implementation, by matching the collaborative network with the functional organization of the enterprise.

\section{Product and process information in SME: dataflow}

It is useful to distinguish among products and processes to develop products. Innovative and competitive products increase enterprise's market share whilst efficient and robust industrial processes allows the enterprise emerging over the competitors. As a consequence, the information on enterprise products and processes, in terms of data and metadata (Table 1), has to be as efficient as possible along the whole product lifecycle. 
Table 1: Enterprise information

\begin{tabular}{|r|c|c|}
\hline & Product data & Processes \\
\hline Business & Metadata (STEP, PDM) & Enterprise content management \\
\hline Engineering & Data (GPS, STEP) & Manufacturing planning \\
\hline
\end{tabular}

PDM/PLM systems were born to control data proliferation in design activities, therefore their primary function is the control of product data in terms of integrity, protection and modification. A second important function regards data availability: user-friendly, simultaneous and multi-point access to product data [20].

PLM systems do not limit their function to database repository, but actively support product data development by tracking design activities, maintaining configuration of evolving products, and allowing the re-use of the data in the development of new products [9].

The enlargement of the scope of product data, which are no more property of design function but become capital of the whole enterprise, requires a redefinition of their accessibility and modifiability, with regard to other IT systems like ERP. The redistribution of the responsibilities on product data among enterprise functions represents the most innovative aspect of PLM systems. Obviously it is also the most difficult aspect to be addressed in PLM implementation and it justifies the lateness of PLM diffusion in the SMEs.

PLM systems have an impact on the entire enterprise and require a structured organization where informative flows, interfaces and decision makers are well identified. This is normal in large companies, but it is not predictable in SMEs, where the same person often provides different functions and where decision makers are individuated more on the basis of capital share than on the basis of organizational role and technical competence.

The intrinsic complexity of PLM systems and the informal organization of small companies represent a real difficulty in the implementation of PLM in SMEs [1].

\subsection{SME organizational model}

The technical literature widely illustrates case studies related to the implementation of PLM in large and well structured organizations. Unfortunately few experiences are available on PLM implementation in SMEs, where informal organizational structure does not allow the application of standard methodologies, based on the development of a detailed ontological model (SAP, UML, ...), in order to perform an efficient description of enterprise processes [8], [17], [25].

A preliminary analysis of enterprise organization should rely on the generic product lifecycle functions [2] schematized in Figure 1:

- Direction: The overall project management, this function is transversal to the others.

- Design concept: Idea for new product or, more often, product design enhancement is recognized, based on market knowledge.

- Market demand analysis: Manufacturer studies need for the new product design and estimates demand and feasibility of meeting demand.

- Engineering: Design engineers create the product design using all available information from the PLM system, including after-market factors, manufacturability data, customers' needs/preferences, and more.

- Sourcing: Procurement carry out preliminary work required to acquire parts, materials, components, equipment, and anything else is required to manufacture the product.

- Production: Product is built to design specifications established by engineering and using parts and materials acquired by sourcing. Conformance to specification is checked through quality control/assurance or process control methodologies.

- Distribution: Product is shipped to either distributors, who store it until order is received from customer, or directly to final customer.

- After market: Products are maintained, serviced, or repaired under warranty or as a value-added service. Using a unique repository of various after-market data ensures that after-market factors are taken into account in subsequent design projects, increasing the value of the product to the customer. 


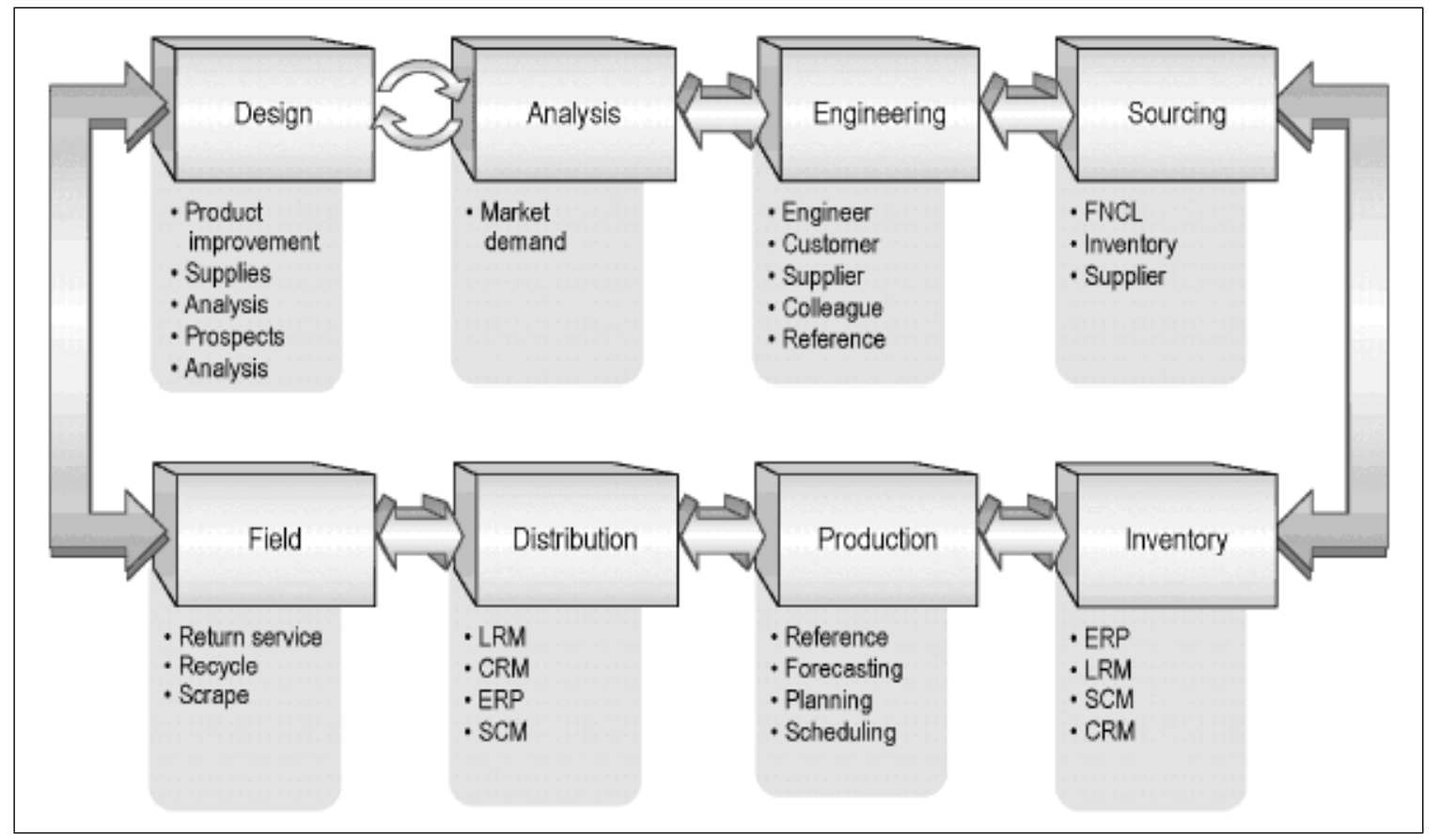

Figure 1: Product lifecycle analysis (from Aberdeen [1]).

The experimental data used in the paper were collected from a SME which designs, manufactures, installs and maintains industrial choppers all around the world. The enterprise structure, according to a functional point of view, adheres to the proposed model.

\subsection{SME investigation procedure}

There is a widespread commonplace saying that every SME works without any notable organization. Actually, the organization exists but it is usually not explicitly formalized: it is a natural organization based on significance relationships and spontaneous collaboration.

Therefore, it makes no sense to look at classical organization devices, like organization charts, information systems, team management tools (PERT, Gantt) [23]. There are four basic principles which make the organization of a SME efficient:

- Process-based cooperation, without a fixed separation of tasks.

- Extended sharing of knowledge, unfortunately transmitted in a informal way.

- Small teams with continuous communications and interchanges of information.

- Potential for outside development by having recourse to the networks of enterprises.

These same principles represent the hardest obstacle to an efficient description of the enterprise data, functions, processes and related managing tools.

Researchers adopt a simple data-driven approach focused on the investigation of enterprise documents in order to overcome the obstacle. A set of questionnaires submitted to enterprise people are used to collect information on produced and consulted documents thus providing an unbiased objective description of the true organization, processes and hierarchies operating in the enterprise.

The questionnaires play a fundamental role in diminishing the noise involuntarily introduced by interviewed people: the focus on documents and their management and not on sensible data regarding the enterprise organization, defines the search field and the required information.

Researchers submitted questionnaires to enterprise personnel during the development of a new project and transformed the enterprise in a living laboratory where researchers interacted with employers during the questionnaires filling, observed the product development process and analyzed the documents identifying their dependence relationships as well as their format. 


\subsection{SME information reorganization}

Several operations applied to the information collected by questionnaires, provide a more structured description of enterprise and its organization, functions, processes and hierarchies:

- Analysis and evaluation of PLM functionalities in the enterprise context;

- Analysis and definition of product data: quantity, relationships, applicability, supporting media;

- Definition of enterprise functions and analysis of their role in product data management;

- Analysis of enterprise's acquaintance with informatics;

- Development of the DLSM (Data Lifecycle Simulation Matrix) representing the lifecycle of product data and their dependencies within the enterprise functions;

- Development of the DISM (Data Interchange Simulation Matrix) representing the interfaces used to communicate the product data among the different functions;

- Creation of a metric to exploit the enterprise position, according to technological and organizational aspects, against the best practice.

The DLSM and DISM matrices provide a complete picture of product data management across the enterprise functions and along the product lifecycle. On this basis it is possible to evaluate the organizational and informational structure by detailing the interactions with each enterprise function. Moreover, analyzing the DLSM and DISM matrix it is possible to extrapolate useful indicators which address the reorganization of the enterprise.

\section{Reorganizing the data: DLSM and DISM Matrices}

The core of the methodology is the building of a DLSM (Data Lifecycle Simulation Matrix), a square matrix containing a complete description of the enterprise organization based on its documents. The DLSM matrix represents the dependence relationship among the data/documents inside different enterprise functions.

The rows of the DLSM matrix, contain the enterprise documents classified according to the enterprise function responsible for their production and maintenance. The columns of the DLSM matrix contain the same documents presented in the same order. The dependence relationships among documents are made explicit by activating the cells at the intersection of the row and of the column corresponding to the associated documents. When a cell is activated, the document along the column will make use/reference to the document identified in the row. A formal description of DLSM matrix is:

$$
\begin{aligned}
\operatorname{DLSM}(i, j=1) & =\{\text { Enterprise documents - Source }\} \\
\operatorname{DLSM}(i=1, j) & =\{\text { Enterprise documents - Access }\} \\
\operatorname{DLSM}(i, j) & = \begin{cases}1 & \text { when document } i \text { is accessed by document } j \\
0 & \text { otherwise }\end{cases}
\end{aligned}
$$

Figure 2 shows a pattern view of the 160x160 sparse DLSM matrix representing the documents required to describe product lifecycle within the analysed case study. The grey areas placed on the matrix diagonal highlight the enterprise functions: Direction, Marketing, Administration, Design, Planning, Supply, Manufacturing and assembling, Inventories, After sale services.

Figure 3 shows the upper and left corner of DLMS matrix including some documents produced by the two functions Direction and Marketing. Some interesting results can be extracted from simple operations performed on the DLSM matrix.

The DISM (Data Interchange Simulation Matrix) is a square matrix having the same structure of DLSM, but now the cells are activated when the format and the support used to save two inter-dependent documents are the same.

The core of the method is the transformation of the DLSM into the DISM. For every intersection activated in the DLSM, the document formats and supports are compared: if they agree (i.e. both MS Word format, or Excel format, or paper support, ...) the cell in the DISM is activated. Obviously there are quite less occurrence in the DISM with respect to DLSM.

Figure 4 represents the upper and left corner of the DISM matrix including some documents produced by the 


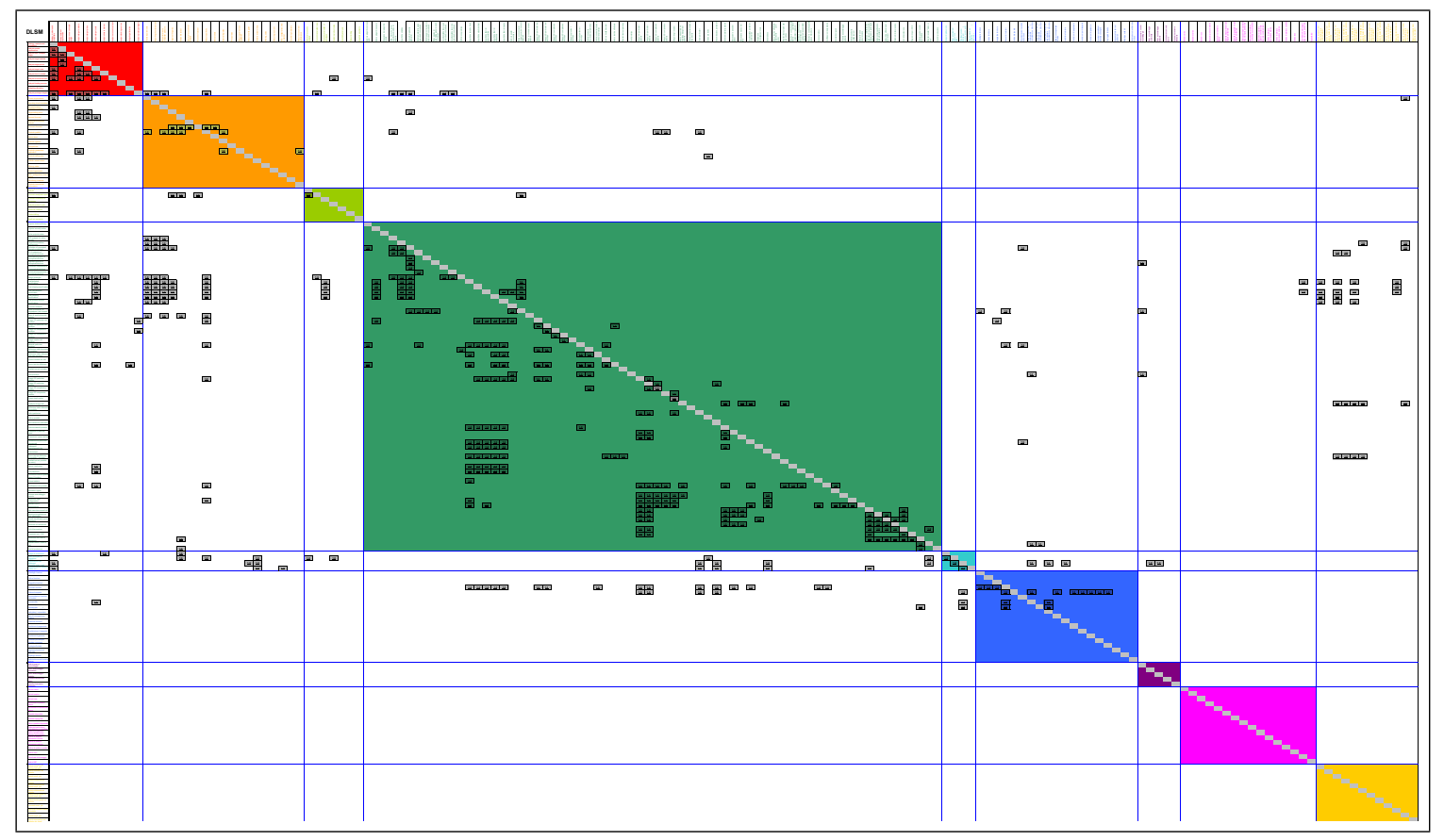

Figure 2: Overview of the complete DLSM.

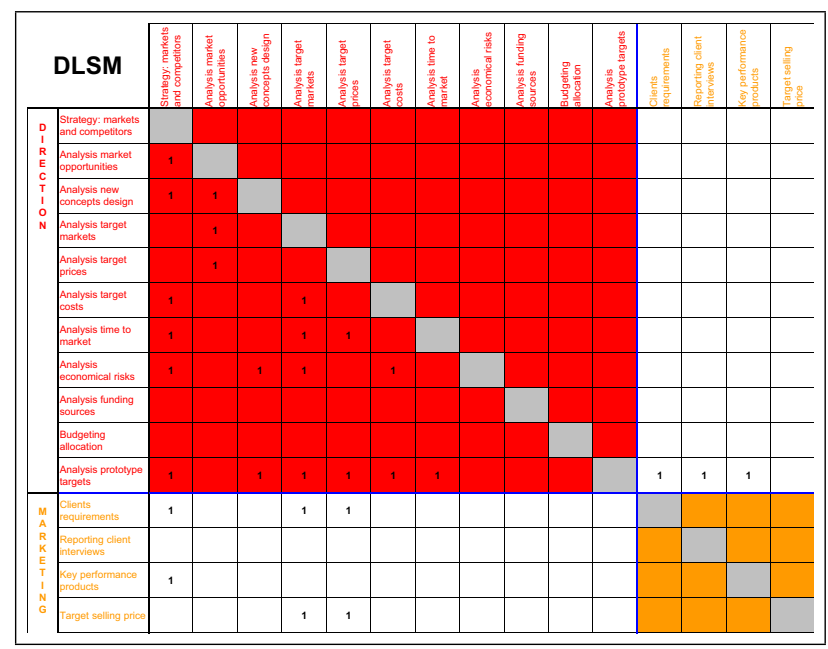

Figure 3: Sample of a portion of DLSM including some documents of the function "Direction". 
enterprise function "Direction". For every intersection activated in the DISM, the compatibility of the adopted applications and their publication on the firm's network is verified and the sum over the rows and the columns occurrence are generated in order to deduce the integration in the product data flow.

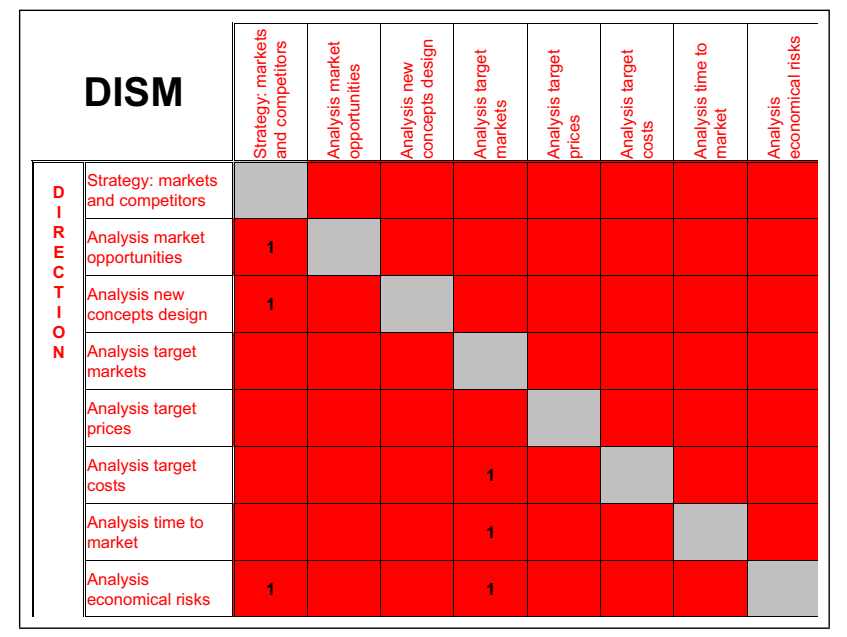

Figure 4: Sample of a portion of DISM including some documents of the function "Direction".

The activated cells in the DISM play a relevant role from a PLM perspective when the document format and the support are electronic. Taking in account only the interfaces based on electronic format, the distance of DISM from DLSM addresses the investments in the informational infrastructure the enterprise needs.

\subsection{Analysing the DLSM matrix}

The sum $\alpha$ and $\beta$ of the occurrences along the rows and the columns of DLSM allows to determine quantitatively the amount of functional dependencies among the documents in the information flow. Table 2 presents the $\alpha$ and $\beta$ values for the document "Analysis target prices" produced by the enterprise function "Direction":

Table 2: Dependencies of "Analysis target prices" document.

\begin{tabular}{|l|c|c|}
\hline Document & $\alpha$ & $\beta$ \\
\hline Price target & 1 & 7 \\
\hline
\end{tabular}

The document is consulted by only another document ("Analysis of market opportunities", produced by the function Direction) and requires the existence of other seven documents belonging to the Direction, Marketing and Design functions.

It is noteworthy that the same document can be shared among different functions inside the enterprise, therefore another important parameter is the percentage of occurrences of every document $(i)$ inside every function $(j)$, associated to the matrix $\operatorname{DLSM}(i, j)$.

As an example Figure 5 shows the percentage use of all the documents generated by the function "Direction". In the collaboration network, the function "Direction" behaves as a supplier, the other enterprise functions are then the clients.

The analysis can be profitably reverted in Figure 6 that shows the percentage use of documents, generated by other functions, through the function "Direction", which now acts as the client for the other functions. Different analyses of DLSM structure bring to light unpredicted outcomes.

\section{Clustering the DLSM: multivariate analysis}

The scope of this section is illustrating the motivations behind the choice of the clustering method used to group the project documents. Clustering of the documents is the main application of the DLSM matrix. Produced documents have already a taxonomy, belonging to one of the product lifecycle functions, that correspond directly 


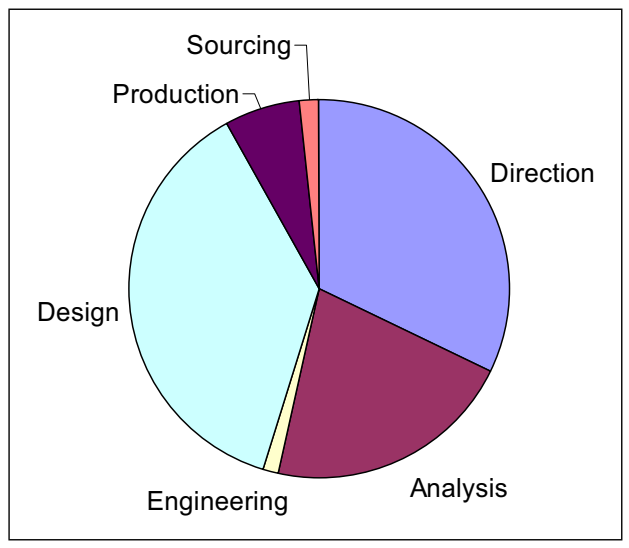

Figure 5: Use of Direction documents by different enterprise functions including Direction (labels represents the destinations).

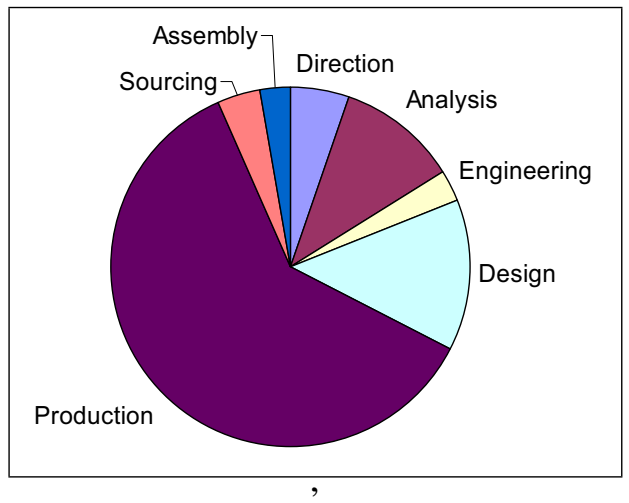

Figure 6: Use of enterprise data by the Direction function (labels represents the origins).

to the enterprise departments. Nevertheless, a typical SME structure is by far different and usually simpler than what appears from the organizational chart. Therefore it is noteworthy to test if the clustering of the document based on their correlations matches the official taxonomy.

We remark that the clustering performed here has nothing to do with the known study field of Information Science that goes under the name of Document Classification. This latter can be defined in the following way [24]: "Document clustering is a fundamental operation used in unsupervised document organization, automatic topic extraction, and information retrieval. It provides a structure for organizing large bodies of text for efficient browsing and searching".

In our case, document clustering, based on the DLSM matrix of correlations, is only a pretext to analyse the enterprise organization from an unbiased point of view. In common with the ordinary Document Classification is the choice of unsupervised clustering methods. As there are reasonable doubts that the real structure of the collaboration network would match the enterprise structure it is convenient not to use the knowledge about the existing organization and leave the classification of the documents to an unsupervised method: the Statistical Cluster Analysis.

Cluster analysis investigates grouping by minimizing a suitable distance measure among the data. The distance function should give a way to measure the similarity between two documents. Euclidean distance is obviously improper for measuring the similarity in the correlations among documents. For the research aims the best suited is the Hamming distance that calculates the percentage of attributes that differ between two objects. Given the DLSM, the element $\operatorname{DLSM}(i, j)$ represents the attribute $j$ of the document $i$. The number of documents and of attributes is the same, $N$. For every two documents $r, s$ the Hamming distance $d_{H}$ is:

$$
d_{H}=\frac{D L S M(r, j)-D L S M(s, j)}{N}
$$


Hierarchical Clustering is the more appropriate technique for working with ordinal metrics. It creates a cluster tree, which is not a single set of clusters, but rather a multi-level hierarchy, where clusters at one level are joined as clusters at the next higher level. The decision of the most appropriate level of clustering is found by choosing the threshold of the inconsistency coefficient for each link of the hierarchical cluster tree. There are different agglomerative techniques [3], among which the nearest neighbour or single linkage. Two clusters are joined together on the basis of the minimum distance between the two nearest elements among all the existing clusters. If cluster $A$ is made of documents $a_{i}(i=1, \ldots, m)$ and cluster $B$ is made of documents $b_{j}(j=1, \ldots, r)$, the distance between the two clusters is:

$$
d_{s l}=\min \left(d_{h}\left(a_{i}, b_{j}\right)\right) \forall i, \forall j
$$

Single linkage is seldom selected among the techniques because it has the drawback of creating elongated cluster (in a spatial visualization of the distance measure) if there is a long chain of documents each one differing from the successive for some attribute. In present study the attributes are the dependencies of documents and it is reasonable to expect that every document be the starting point for a successive document.

Before to apply the clustering, a pre-processing has been executed on the DLSM to reduce the search space [5]. After a binary sorting, the void rows and columns have been excluded from the clustering. These represent documents which have been produced independently the ones from all the others. It is possible that most of them be useless documents and be requested by internal bureaucratic procedures.

The result of the application of the hierarchical clustering to the modified DLSM is the dendrogram graph represented in Figure 7. The dashed line represents a threshold inconsistency value chosen to group the documents in ten clusters which correspond to the nine divisions actually present in the enterprise.

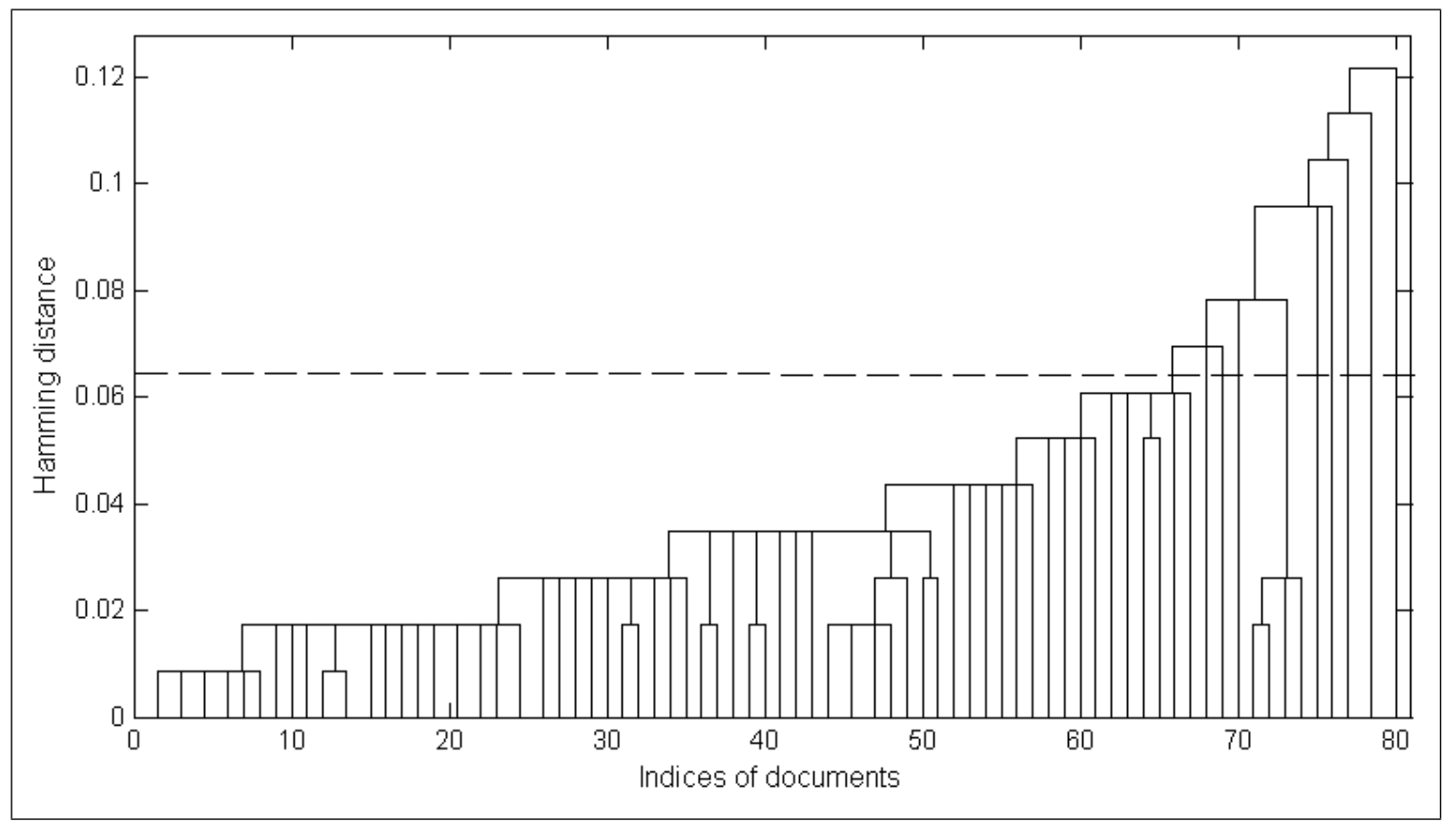

Figure 7: Dendrogram of the hierarchical tree for the DLSM matrix.

A number of considerations can be drawn on the result of this clusterization:

- One cluster (document index from 1 to 67) is by far larger than the others and includes documents belonging to nearly all the divisions;

- With the exception of the fourth cluster (document index from 71 to 74 ), all the others have only one document inside;

- These one-document clusters have an high unconsistency value, therefore they cannot be attributed to a larger one changing the agglomerative technique.

The first consideration describe a positive situation. The majority of documents have strict reciprocal dependencies. A possible explanation is that the SME applies implicitly a concurrent engineering strategy in the 
management of new projects. As a matter of fact every project is carried on by an unstructured working team made usually of few persons if not just one, who are responsible for the production of the key documents which are used by all the divisions to produce their own documents.

Second and third considerations indicate the presence of dead end documents, i.e. documents that get their data from other documents but are no longer used to produce new documents. This is not necessarily an issue as it seems natural that every division produces a final report on the project done. The fourth cluster instead represents a small number of documents totally independent from the others. The issue is not in the presence of a cluster but in the high value of the inconsistency index (measured by the length of the dendrogram branches) that denotes an excessive distance between this cluster and every other.

From a PLM perspective the results of the DLSM clustering suggests the need of investments in the sharing of documents. Under the hypothesis of a working team operating in a concurrent engineering framework is essential to increase the accessibility of documents by all the enterprise functions.

In a SME, the issues related to the condivision of product data are strictly related to the possibility of access to the different database and data formats. Inside the large majority of the SMEs, the integration of the enterprise documentation is not considered a critical issue [11].

\section{Evaluating the attitude to change}

The questionnaires provide a picture of product data management across the enterprise functions and along the product lifecycle. Such information can be reorganized according to the DISM in order to gain a better knowledge on enterprise organization. On this base it is possible to evaluate the impact of PLM on the informational structure by detailing the effects on each enterprise function.

The evaluation of attitude to collaborative working in the CE context should be expressed in terms of costs and benefits, which depend on enterprise readiness and propensity to accept innovation [19]. The test case focused on the analysis of two attitude indexes: attitude to organizational changes and attitude to informational changes.

The attitude to innovation assumes different characteristics depending on enterprise functions. Authors extracted from technical literature a set of criteria to be applied to the most relevant functions, as identified on the basis of DSLM and DISM. Different evaluating scales and different weights have been applied according to criteria nature [17].

The results of evaluation of both organizational and informational indexes for the most relevant enterprise functions (P1 - Marketing, P2 - Design, and P3 - Supply) are summarized in Figure 8, which represents a pictorial description of the enterprise global attitude to innovation.

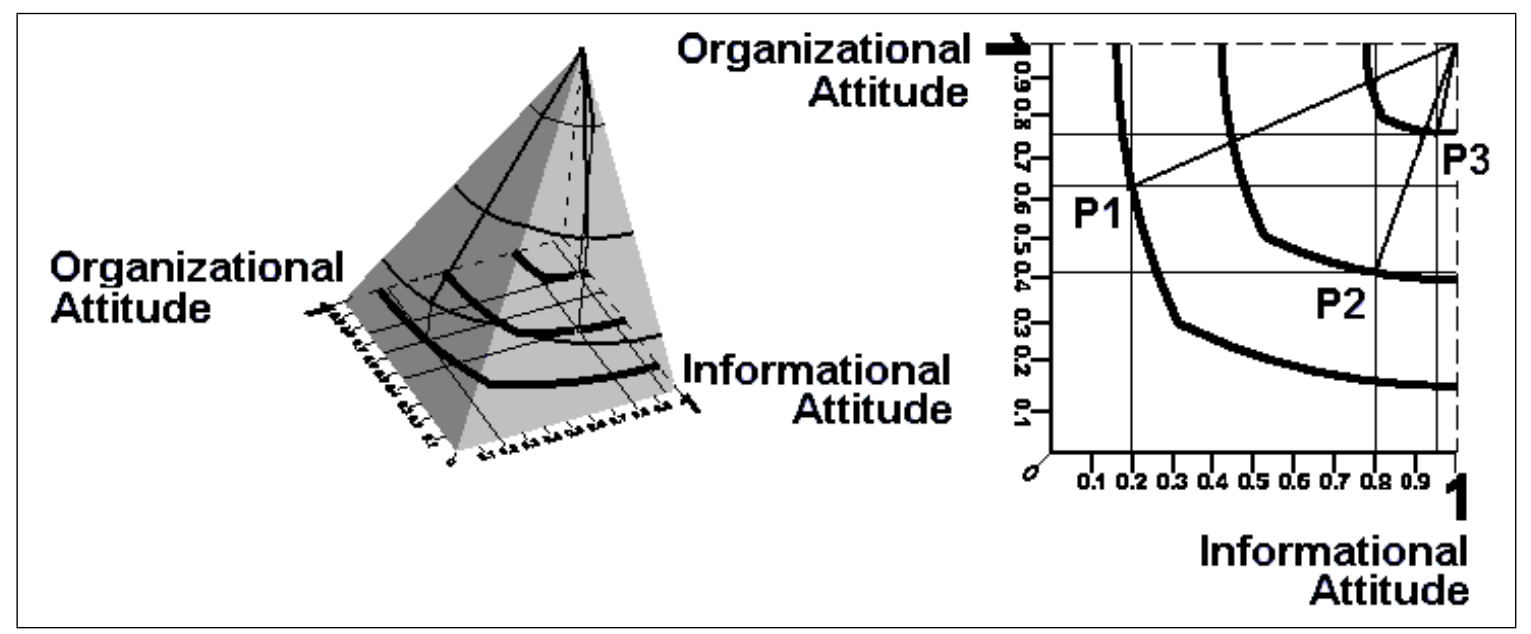

Figure 8: Global attitude index for the test case. 


\section{Conclusions}

The paper focuses on the development of a method for the evaluation of enterprise readiness to collaborative product and process design, with particular attention to the implementation of PLM in the SMEs.

Data collection relies on questionnaires focused on the document management process adopted in the enterprise. The questionnaires are submitted to enterprise personnel while researchers integrates knowledge and analysis of decisional processes.

In order to evaluate the amount of Business Process Management required to upgrade the enterprise organisation to PLM pre-requisites, the authors developed an analysis tool consisting of a classification of product data flows and their supporting formats. Subsequent refinements of the gathered information allow to benchmark the enterprise propensity to the implementation of PLM.

The simple approach proposed in the paper seems to solve some of the problems related to the introduction of information technology systems in SMEs: insufficient awareness of organizational issues, insufficient involvement of end users, inadequate traininig of users.

\section{Bibliography}

[1] Aberdeen, "PLM - It's for Any Manufacturer Striving for Product Excellence", An Executive White Paper, Aberdeen Group, Inc., www.aberdeen.com, 2002.

[2] R.W. Bagshaw, S.T. Newman, "Structured approach to the design of a production data analysis facility Part 1: Conceptual design and information requirements", Int. J. Prod. Res., Vol. 39, no. 10, pp. 2121-2141, 2001.

[3] A. Cevahir, "Clustering of Correlated Documents into Designated number of Clusters: A Practical Approach", www.cs.bilkent.edu.tr, 2005.

[4] D. Chen, G. Doumeingts, "Developing a Theory of Design - A Tentative Approach Using Mathematics", Studies in Informatics and Control, Vol.11, No.1, 2002, ICI, Bucharest.

[5] G. Czibula, G. Serban, "Hierarchical Clustering Based Design Patterns Identification", Int. J. of Computers, Communications \& Control, Vol. III, pp. 248-252, 2008, CCC Publications.

[6] Datamation Limited, "Understanding Product Lifecycle Management", Rep. No. PLM-11, Rev. No. 1.0, September 2002, Cambridge, UK.

[7] European Commission, SME and cooperation, Observatory of European SMEs, 2003, Enterprise Pubblication, Brussels.

[8] B. Eynard, T. Gallet, P. Nowak, L. Roucoules, "UML based specifications of PDM product structure and workflow", Computers in Industry, Vol.55, pp. 301-316, 2004.

[9] B. Fagerström, L. Olsson, "Knowledge Management in Collaborative Product Development", Systems Engineering, Vol. 5, No. 4, pp. 274-285, 2002.

[10] F.G. Filip, "Decision support and control for large-scale complex systems", Annual Reviews in Control, Vol.32, No.1, pp. 61-70, 2008.

[11] F. Franceschini, Advanced quality function deployment, Boca Raton, St. Lucie Press, 2002.

[12] H. Kaschel, L.M. Sánchez y Bernal, "Importance of Flexibility in Manufacturing Systems", Int. J. of Computers, Communications \& Control, Vol. I (2), pp. 53-60, 2006, CCC Publications.

[13] J. Krauth, Introducing information technology in small and medium sized enterprises, Studies in Informatics and Control, Vol.8, No.1, 1999, ICI, Bucharest.

[14] A. Kusiak, Concurrent Engineering: Automation, Tools, and Techniques, Wiley, USA, 1992.

[15] T.W. Malone, K. Crowston, "The interdisciplinary study of coordination", ACM Computing Surveys (CSUR), Vol. 26, No. 1, pp.87-119, 1994.

[16] S.Y. Nof, G. Morel, L. Monostori, A. Molina, F. Filip, "From plant and logistisc control to multi-enterprise collaboration", Annual Reviews in Control, Vol.30, No.1, pp. 55-68, 2006. 
[17] K. Schmid, I. John, "A customizable approach to full lifecycle variability management", Science of Computer Programming, Vol.54, pp. 259-284, 2004.

[18] M.G. Singh, A. Titli, Systems Decomposition, Optimisation and Control, Pergamon Press, 1988.

[19] R. Sudarsan, S.J. Fenves, R.D. Sriram, F. Wang, "A product information modelling framework for product lifecycle management", Computer Aided Design, Vol.37, pp.1399-1411, 2005.

[20] J. Stark, Product LifeCycle Management - 21st Century Paradigm for Product Realisation, 2005, SpringerVerlag, London, UK.

[21] S.N. Talukdar, "Collaboration rules for autonomous software agents", Decision Support Systems, Vol. 24, pp.269-278, 1999.

[22] S. Tichkiewitch, D. Brissaud, "Co-ordination Between Product and Process Definitions in a Concurrent Engineering Environment", Annals of CIRP, Vol. 49, pp. 75-78, 2000.

[23] A. Tiwana, B. Ramesh, "A design knowledge management system to support collaborative information product evolution", Decision Support System, Vol.31, pp.241-262, 2005.

[24] C. Xiaohui, G. Jinzhu, T.E. Potok, "A flocking based algorithm for document clustering analysis", J. of Systems Architecture, Vol.52, pp. 505-515, 2006, Elsevier.

[25] S. Zina, M. Lombard, L. Lossent, C. Henriot, "Generic Modeling and Configuration Management in Product Lifecycle Management", Int. J. of Computers, Communications \& Control, Vol. I (4), pp. 126-138, 2006, CCC Publications.

Dario Antonelli Italy 1966, holds a M.S. Degree in Mechanical Engineering from the Politecnico di Torino, 1990. He worked at Fiat Research Center until 1992. He is currently Associate Professor at the Department of Production Systems and Economics of Politecnico di Torino. His scientific activity is mainly related to numeric Finite Element Simulation of metalworking processes, to experimental identification of process parameters and to Supply Chain Management.

Paolo Chiabert (b. June 28, 1963). M.Sc. Degree in Electronic Engineering, Politecnico di Torino (1993), IBM Consultant (1994), Researcher in Design and Methods of Industrial Engineering at the Politecnico di Torino (1995-2003), Associate Professor in Technology and Manufacturing Systems (2004). According to the Product Lifecycle Management perspective, the research interests range from Design methods, to Manufacturing technologies, to Production planning and control, to Quality management. Since 1997 Italian expert in the ISO Technical Committee 213 - Geometrical Product Specifications and Verification, he is member of the GPS Technical commission at UNI. 